\title{
Antropometría, Composición Corporal y Somatotipo de las Patinadoras de Elite en Patinaje Artístico sobre Ruedas: Análisis por Disciplinas
}

\author{
Anthropometry, Body Composition and Somatotype Characteristics \\ of World Class Female Roller Skaters by Discipline
}

María Helena Vila Suárez; ; Carmen Manchado López ${ }^{* * *}$ \& Carmen Ferragut Fiol***

VILA, M. H.; MANCHADO, C. \& FERRAGUT, C. Antropometría, composición corporal y somatotipo de las patinadoras de elite en patinaje artístico sobre ruedas: análisis por disciplinas. Int. J. Morphol., 33(3):1130-1135, 2015.

RESUMEN: El objetivo de este estudio fue describir y comparar las características antropométricas, composición corporal y somatotipo de patinadoras de patinaje artístico sobre ruedas por categorías (junior y senior) y por disciplinas (individual, parejas y danza). Fueron evaluadas 62 patinadoras profesionales (36 en la disciplina de libre, 13 en danza y 13 en parejas). El análisis estadístico utilizado fue una ANOVA para muestras independientes entre disciplinas, y una T de Student para las categorías (junior y senior). Entre la categoría junior y senior no se han encontrado diferencias estadísticas significativas. Las patinadoras junior presentaron un somatotipo endo-mesomorfo, y las patinadoras senior presentaron un somatotipo mesomorfo balanceado. El somatotipo de las patinadoras de parejas y danza fue el mesomórfico balanceado, y el de las patinadoras de libre fue endo-mesomórfico. Este estudio aporta datos biotipológicos de referencia para la población de elite de patinadoras sobre ruedas.

PALABRAS CLAVE: Mujeres; Elite; Disciplina danza; Disciplina individual; Disciplina parejas; Porcentaje graso.

\section{INTRODUCCIÓN}

Diferentes estudios sugieren que cada deporte se caracteriza por unas características morfológicas específicas que favorecen el llegar al alto rendimiento, e incluso para cada modalidad deportiva pueden existir características específicas (Leone et al., 2002; Norton \& Olds, 2001; Ross et al., 1977; Mosnma \& Malina, 2005). La relevancia de las características antropométricas y la composición corporal son especialmente importantes en los deportes considerados "artísticos", como la gimnasia y el patinaje artístico sobre ruedas o sobre hielo.

El patinaje artístico sobre ruedas es una especialidad deportiva, que engloba cuatro disciplinas distintas: figuras, libre, parejas y danza. Excepto la disciplina de figuras, las otras tres disciplinas tienen en común que durante la competición son puntuadas por jueces en función de la dificultad y ejecución de las habilidades realizadas. Sin embargo, también inciden en las puntuaciones el aspecto estético (capacidad de comunicarse interpretación artística de la música a través del movimiento humano). De este modo, el ren- dimiento depende en gran medida de una evaluación o impresión subjetiva de las formas del cuerpo que se suma al mérito artístico en la puntuación final emitida por los jueces (Monsma \& Malina; Ross et al.; Vadocz et al., 2002; Whisenhunt et al., 2008).

La producción científica sobre el Patinaje Artístico sobre ruedas es escasa, y prácticamente inexistente sobre aspectos de la estructura corporal y las mujeres patinadoras.

Por ello las referencias aquí presentadas versan sobre la disciplina de patinaje sobre hielo. Las patinadoras de elite tienden a ser pequeñas, delgadas y ligeras; y necesitan de unas capacidades físicas específicas (flexibilidad, fuerza explosiva, coordinación y equilibrio) (Ross et al.; Niinimaa, 1982; Mosnma \& Malina; Vadocz et al.; Brown, 2001). La tendencia hacia bajas estaturas y pesos ligeros podría, en parte, ser atribuida a que la linealidad beneficia el momento de inercia requerido, aspecto que permite realizar a las patinadoras saltos con giros y acrobacias de mayor dificul-

\footnotetext{
* Faculty of Education and Sport Science, University of Vigo, Pontevedra, Spain

** Faculty of Education, University of Alicante, Alicante, Spain.

**** Faculty of Medicine, University of Alcalá, Alcalá de Henares, Spain.
} 
tad (Niinimaa). En este deporte, las características de linealidad están incluidas con los ideales de delgadez socioculturales que están presentes entre los entrenadores, cuerpo técnico y jueces (Monsma, 2008).

Constatada la escasa investigación realizada sobre el patinaje artístico sobre ruedas, se ha realizado la siguiente investigación, la cual pretende desarrollar dos grandes objetivos. El primero fue, describir y comparar las características antropométricas, composición corporal y somatotipo de patinadoras de elite por disciplinas deportivas (individual, parejas y danza). En segundo objetivo, establecer si existen diferencias en las características antropométricas, composición corporal y el somatotipo de patinadoras de elite por categorías (junior y senior).

\section{MATERIAL Y MÉTODO}

Sesenta y dos patinadoras participantes en el 51st Campeonato del Mundo de Patinaje Artístico sobre Ruedas. De las cuales 24 patinadoras pertenecen a la categoría junior y 38 a la categoría senior. Por disciplinas, 36 participaron en la disciplina de individual, 13 en parejas y 13 en danza. Las patinadoras participantes de este estudio 28 son europeas, 14 Americanas y 20 suramericanas.

Para el desarrollo de la investigación se obtuvo la autorización de la Real Federación Española de Patinaje, de la Federación Internacional y del Comité de Organización del Campeonato del Mundo. Días previos a la competición, se contactó a través de la organización del Campeonato con las diferentes selecciones nacionales y se informó a los diferentes equipos técnicos de los países participantes, así como a todos los deportistas, de los objetivos del estudio, los riesgos y beneficios de la investigación. Todos los deportistas firmaron un consentimiento informado sobre la valoración llevada a cabo, o su representante legal si éste era menor de 18 años de edad. El estudio se llevó a cabo de acuerdo con la Declaración de Helsinki.

La valoración antropométrica ha seguido las normas, técnicas de medida y criterios recomendados por la International Society for the Advancement of Kinanthropometry (ISAK) (Stewart et al., 2011). Las variables registradas fueron: masa corporal, talla, envergadura, 6 pliegues cutáneos (subescapular, tricipital, cresta ilíaca, abdominal, anterior del muslo y pierna), 3 diámetros (bicondíleo de fémur, biepicondíleo del húmero y biestiloideo de la muñeca), y 9 perímetros (brazo relajado, brazo flexionado y contraído, antebrazo (perímetro máximo), muñeca, glúteos, superior del muslo, medial del mus- lo, pantorrilla y tobillo). Todas las medidas bilaterales fueron en el lado derecho. Los pliegues cutáneos se tomaron en el lado derecho, dando la media de tres medidas. Los antropometristas fueron cuatro, tres estaban acreditados con el nivel 1 y uno con el nivel 2 de la ISAK. En todos los evaluadores, su error técnico de medida se sitúa dentro de los rangos recomendados. El instrumental fue calibrado con antelación para evitar errores en la medición.

El instrumental utilizado se componía de: 1) Una báscula con tallímetro, marca SECA® (SECA, Alemania) de escala de $0.1 \mathrm{~kg}$ para el peso y $1 \mathrm{~mm}$ para la altura. 2) Un plicómetro Holtain ${ }^{\circledR}$ de $2 \mathrm{~mm}$ de precisión (Holtain Ltd., Reino Unido), para registrar los pliegues cutáneos. 3) Un paquímetro Holtain ${ }^{\circledR}$ de $1 \mathrm{~mm}$ de precisión (Holtain Ltd., Reino Unido) con el que se determinaban los diámetros. 4) Una cinta métrica inextensible para los perímetros (Lufkin Executive Thinline, W606PM, USA), de $1 \mathrm{~mm}$ de precisión.

Se determinó el índice de masa corporal (IMC) por medio del cociente peso/altura ${ }^{2}$, siendo el peso expresado en kilogramos $(\mathrm{kg})$ y la altura en metros $(\mathrm{m})$. Se estimaron los porcentajes de grasa a partir de las ecuaciones de Faulkner (1986) y Carter (1982). Los tres componentes del somatotipo se determinaron por el método Carter (Carter \& Heath, 1990) y también se determinó el índice de dispersión del somatotipo (SDI) (Carter, 1970).

Los datos se recogieron en una base de datos " $a d$ hoc" de Excel (Microsoft Office ${ }^{\circledR}$ v.2003), el tratamiento estadístico se realizó con el programa SPSS® v.15.0 (para Windows). Se analizó la distribución de probabilidad de las distintas variables de estudio mediante el cálculo de estadísticos descriptivos básicos (media, desviación típica), y la realización de tests de hipótesis (prueba de KolgomorovSmirnov y Lilliefors) y prueba de homogeneidad de Levene. Para establecer las posibles diferencias entre categorías se realizó una comparación de medias a través de la prueba T de Student para muestras independientes. Para establecer las posibles diferencias entre disciplinas fue utilizado el análisis de la varianza (ANOVA). Cuando se comprobó la existencia de diferencia estadística significativa, se utilizó la prueba de Tukey-Kramer (Kramer, 1956). Se consideraron significativas aquellas diferencias o resultados estadísticos cuya probabilidad por azar fue superior al $95 \%(\mathrm{p}<0,05)$.

\section{RESULTADOS}

Los valores medios de las patinadoras por categorías júnior y senior se presentan en la Tabla I. 
Tabla I. Media y desviación típica correspondiente a las características antropométricas, composición corporal y somatotipo de patinadoras en función de la categoría.

\begin{tabular}{|c|c|c|c|c|}
\hline \multicolumn{2}{|l|}{ Variables } & Sig & Júnior (24) & Senior(38) \\
\hline \multicolumn{2}{|l|}{ Edad (años) } & --- & $16,7 \pm 1,5$ & $22,3 \pm 3,6$ \\
\hline \multicolumn{2}{|l|}{ Talla $(\mathrm{cm})$} & ns & $160,2 \pm 5,3$ & $161,3 \pm 6,6$ \\
\hline \multicolumn{2}{|l|}{ Masa corporal $(\mathrm{Kg})$} & ns & $54,3 \pm 7,7$ & $56,7 \pm 8,0$ \\
\hline \multicolumn{2}{|l|}{ Envergadura $(\mathrm{cm})$} & ns & $161,0 \pm 6,6$ & $162,3 \pm 8,1$ \\
\hline \multicolumn{2}{|l|}{$\operatorname{IMC}(\%)$} & ns & $21,0 \pm 1,9$ & $21,7 \pm 1,9$ \\
\hline \multicolumn{2}{|l|}{ Faulkner $(\%)$} & ns & $17,8 \pm 2,8$ & $17,2 \pm 2,0$ \\
\hline \multicolumn{2}{|l|}{ Carter $(\%)$} & ns & $10,8 \pm 2,0$ & $10,4 \pm 1,5$ \\
\hline \multirow[t]{9}{*}{ Perímetros (cm) } & Perímetro del brazo (relajado) & ns & $26,3 \pm 2,7$ & $26,8 \pm 3,4$ \\
\hline & Perímetro del brazo (flexionado y contraído) & ns & $27,6 \pm 2,7$ & $27,6 \pm 5,0$ \\
\hline & Perímetro del antebrazo (máximo) & ns & $23,7 \pm 2,2$ & $24,0 \pm 3,7$ \\
\hline & Perímetro de la muñeca & ns & $15,4 \pm 1,2$ & $16,1 \pm 2,4$ \\
\hline & Perímetro del glúteo & ns & $92,9 \pm 7,1$ & $91,8 \pm 11,2$ \\
\hline & Perímetro del muslo (superior) & ns & $56,0 \pm 4,0$ & $55,4 \pm 3,8$ \\
\hline & Perímetro del muslo (medial) & ns & $50,6 \pm 3,6$ & $51,0 \pm 3,8$ \\
\hline & Perímetro de la pantorrilla (máximo) & ns & $35,0 \pm 2,4$ & $34,7 \pm 2,3$ \\
\hline & Perímetro del tobillo & ns & $23,0 \pm 4,8$ & $22,4 \pm 1,6$ \\
\hline \multirow[t]{3}{*}{ Diámetros (cm) } & Biestiloideo & $* *$ & $4,9 \pm 0,6$ & $5,4 \pm 0,8$ \\
\hline & Biepicondileo del húmero & ns & $6,2 \pm 0,7$ & $6,5 \pm 0,6$ \\
\hline & Bicondíleo del fémur & ns & $9,4 \pm 1,2$ & $9,8 \pm 1,6$ \\
\hline \multirow[t]{4}{*}{ Somatotipo } & Endomorfismo & ns & $3,3 \pm 1,2$ & $3,0 \pm 1,1$ \\
\hline & Mesomorfismo & ns & $4,2 \pm 1,0$ & $4,0 \pm 1,0$ \\
\hline & Ectomorfismo & ns & $2,7 \pm 0,9$ & $3,0 \pm 1,1$ \\
\hline & SDI & --- & $3,0 \pm 1,5$ & $3,5 \pm 2,0$ \\
\hline
\end{tabular}

$\mathrm{p}<0,001$.

Tabla II. Media y desviación típica correspondiente a las características antropométricas, composición corporal y somatotipo de patinadoras en función de la disciplina.

\begin{tabular}{|c|c|c|c|c|c|}
\hline \multicolumn{2}{|l|}{ Variables } & Sig & In divid ual (36) & Parejas (13) & Danza (13) \\
\hline \multicolumn{2}{|l|}{ Edad (años) } & --- & $20,1 \pm 4,1$ & $19,2 \pm 3,6$ & $20,8 \pm 4,4$ \\
\hline \multicolumn{2}{|l|}{ Talla $(\mathrm{cm})$} & $\mathrm{a}^{* * *}, \mathrm{c} * *$ & $166,4 \pm 6,2$ & $156,7 \pm 3,0$ & $163,4 \pm 6,5$ \\
\hline \multicolumn{2}{|l|}{ Masa corporal (Kg) } & --- & $54,7 \pm 8,3$ & $51,3 \pm 5,4$ & $57,3 \pm 7,9$ \\
\hline \multicolumn{2}{|l|}{ Envergadura (cm) } & $\mathrm{a}^{* * *}, \mathrm{c}^{* * *}$ & $162,7 \pm 6,4$ & $155,8 \pm 6,4$ & $165,4 \pm 8,7$ \\
\hline \multicolumn{2}{|l|}{$\operatorname{IMC}\left(\mathrm{Kg} / \mathrm{m}^{2}\right)$} & --- & $21,7 \pm 2,0$ & $20,8 \pm 1,7$ & $21,4 \pm 1,8$ \\
\hline \multicolumn{2}{|l|}{ Faulkner (\%) } & --- & $17,5 \pm 2,3$ & $17,7 \pm 3,0$ & $17,1 \pm 1,8$ \\
\hline \multicolumn{2}{|l|}{ Carter (\%) } & --- & $10,5 \pm 1,7$ & $10,7 \pm 2,2$ & $10,2 \pm 1,3$ \\
\hline \multirow[t]{9}{*}{ Perímetros (cm) } & Perím etro del brazo (relajado) & $\mathrm{b}^{* * *}$ & $25,6 \pm 2,6$ & $26,9 \pm 3,1$ & $29,2 \pm 3,2$ \\
\hline & Perím etro del brazo (flexionado y contraído) & --- & $27,1 \pm 2,5$ & $28,1 \pm 2,8$ & $28,7 \pm 8,1$ \\
\hline & Perím etro del antebrazo (máximo) & --- & $23,4 \pm 2,5$ & $24,6 \pm 1,9$ & $24,5 \pm 6,1$ \\
\hline & Perím etro de la muñeca & --- & $15,2 \pm 1,0$ & $16,1 \pm 1,2$ & $17,5 \pm 3,7$ \\
\hline & Perímetro del glúteo & $\mathrm{c}^{*}$ & $92,3 \pm 6,3$ & $86,9 \pm 13,3$ & $97,1 \pm 11,9$ \\
\hline & Perím etro del muslo (superior) & --- & $55,6 \pm 3,9$ & $55,1 \pm 4,1$ & $56,3 \pm 3,8$ \\
\hline & Perím etro del muslo (medial) & $a^{*}, c^{*}$ & $50,3 \pm 3,5$ & $49,9 \pm 3,7$ & $53,2 \pm 3,4$ \\
\hline & Perím etro de la pant orrilla (máximo) & --- & $34,6 \pm 2,4$ & $34,9 \pm 2,6$ & $35,2 \pm 2,0$ \\
\hline & Perím etro del tobillo $(\mathrm{cm})$ & --- & $22,6 \pm 4,0$ & $22,9 \pm 1,5$ & $22,3 \pm 1,8$ \\
\hline \multirow[t]{3}{*}{ Diámetros (cm) } & Biestiloideo & $a^{*}, b^{*}$ & $4,9 \pm 0,5$ & $5,6 \pm 1,3$ & $5,6 \pm 0,6$ \\
\hline & Biepicondileo del húmero & --- & $6,3 \pm 0,7$ & $6,5 \pm 0,5$ & $6,8 \pm 0,6$ \\
\hline & Bicondíleo del fémur & --- & $9,4 \pm 1,2$ & $9,9 \pm 2,0$ & $10,1 \pm 1,4$ \\
\hline \multirow[t]{4}{*}{ Somatotipo } & Endomorfismo & $b^{*}$ & $3,4 \pm 1,1$ & $3,0 \pm 1,2$ & $2,5 \pm 0,9$ \\
\hline & Mesomorfismo & --- & $4,0 \pm 1,1$ & $4,1 \pm 0,7$ & $4,4 \pm 1,1$ \\
\hline & Ectomorfismo & --- & $2,8 \pm 1,0$ & $2,9 \pm 1,0$ & $3,0 \pm 1,0$ \\
\hline & SDI & --- & $3,4 \pm 1,7$ & $2,6 \pm 3,8$ & $2,2 \pm 3,3$ \\
\hline
\end{tabular}

* $=\mathrm{p}<0,05 ; * *=\mathrm{p}<0,001 ; \mathrm{a}=$ diferencias individual con parejas; $\mathrm{b}=$ diferencias individual con danza; $\mathrm{c}=$ diferencias parejas con danza. 
De todas las variables analizadas, se han encontrado diferencias estadísticas en el diámetro biestiloideo entre la categoría júnior y senior. Las patinadoras junior presentan un somatotipo endo-mesomorfo, y las patinadoras senior presentan un somatotipo mesomorfo balanceado. Ambas categorías presentaron un índice de dispersión del somatotipo (SDI), entendido como la dispersión de los somatotipos individuales respecto al medio de 3,0 en la categoría júnior y de 3,5 para la categoría senior, reflejando heterogeneidad en ambas categorías.

Se han encontrado diferencias significativas entre la disciplina de individual con la disciplina de parejas en las variables talla, envergadura, perímetro medial del muslo y diámetro biestiloideo. Entre la disciplina de individual con danza se encontraron diferencias estadísticas en el brazo relajado en extensión, en el diámetro biestiloideo y en la endomorfia. Para la talla, envergadura, perímetro del glúteo y medial del muslo se encontraron diferencias estadísticas entre la disciplina de parejas con danza (Tabla II). El somatotipo de las patinadoras de las disciplinas de parejas y danza es el mesomórfico balanceado, para la disciplina de individual es endo-mesomórfico. El SDI indica diferencias significativas en todas las disciplinas (Tabla II).

\section{DISCUSIÓN}

Los resultados de esta investigación indican que las patinadoras de categoría junior y senior presentan similares características antropométricas y de composición corporal. Entre disciplinas, se han encontrado diferencias ente las patinadoras de danza respecto con las patinadoras individuales y de parejas. Se confirma la importancia de la delgadez y linealidad para el patinaje artístico sobre ruedas, y más para las disciplinas de dúo (parejas y danza).

En el análisis por categorías, los resultados confirman lo expresado por Carter \& Heath, estamos ante un deporte en el que las características antropométricas (somáticas) de las deportistas jóvenes son coincidentes con los de las atletas adultas del mismo deporte, puesto que sólo se ha encontrado diferencias significativas en el diámetro biestiloideo. O lo que es lo mismo, se busca la misma imagen corporal independiente de la edad, eso "obliga" a las patinadoras senior a mantener una imagen más acorde a la edad junior (Delistraty et al., 1992; Leone et al.).

La media de edad, peso y talla de las patinadoras senior de esta investigación fue mayor que la de otros estudios publicados (Monsma \& Malina; Ross et al.; Ziegler et al., 2001; Delistraty et al.; Leone et al.). Destacar que a pe- sar de no encontrar diferencias en los valores del IMC, ni en los porcentajes grasos analizados, los valores de IMC encontrados en las categorías junior y senior (21,0 y 21,7 respectivamente) fueron superiores que los presentados por otros estudios (Monsma \& Malina; Ziegler et al.) (20,5 y 19,3 respectivamente).

El comportamiento de la endomorfia es el esperado, a mayor edad los valores de endomorfia en la mujer aumentan (Malina et al., 2004; Ross et al.). Este comportamiento coincide con el estudio de Monsma \& Malina, en su caso, los valores de la endomorfia los relacionan a diferentes técnicas de medición, a la variabilidad de las muestras o a la diferencia de años que han pasado entre los datos (secular trend) de sus estudios. En un deporte donde el aspecto físico-estético es tan importante (Monsma \& Malina; Vadocz et al.; Whisenhunt et al.), se podía esperar que los valores de ectomorfia fueran superiores a los de la endomorfia, como se expone en otros estudios (Malina et al., 2004; Monsma \& Malina; Ross et al.; Vadocz et al.). Puede influir en este resultado que se han valorado a un elevado número de patinadoras, de diferentes niveles desde el punto de vista del rendimiento, aunque todos son participantes en un Campeonato del Mundo, por lo tanto pertenecen a la élite.

A pesar que no se han encontrado diferencias en las características antropométricas (excepto diámetro biestiloideo) ni en la composición corporal, la distancia de dispersión de los somatotipos medios (SDI) indica que existen diferencias estadísticas entre ambas categorías.

El presente estudio consideró realizar un análisis por disciplinas, individual, parejas y danza. Los resultados de este estudio sugieren que para la disciplina de parejas, la poca estatura y la envergadura son un criterio de selección, basado en ventajas biomecánicas (gran estabilidad, incremento del índice fuerza/peso, descenso de los momentos de inercia), pues constituyen un grupo de características que favorecen el manejo de la patinadora en los giros, enlaces, elevaciones y desplazamientos por parte de los patinadores masculinos a la hora de realizar acrobacias (Podolsky et al., 1990; Ziegler et al.; Vadocz et al.). Estos resultados concuerdan con otros estudios realizados en patinaje artístico sobre hielo (Ross et al.; Niinimaa; Monsma \& Malina).

Las diferencias presentadas entre la disciplina de danza respecto a las otras dos disciplinas, son acordes a las características reglamentarias (las patinadoras de danza no pueden realizar elevaciones ni giros aéreos), su rendimiento está enfocado en el aspecto estético con la pareja, por lo que sus características morfológicas pueden mejorar su mérito artístico (Monsma \& Malina). También se valora la similitud en el estilo del patinaje, la coordinación, pues se les exi- 
ge mantener unos desplazamientos paralelos y profundos respecto al patinador masculino (Monsma \& Malina). Para las disciplinas de libre y parejas, las habilidades aéreas son obligatorias, en el caso de las parejas es el patinador el encargado de "lanzar" a la patinadora. Estamos ante tres disciplinas diferentes con exigencias diferentes. Estos resultados coinciden con el estudio presentado por Monsma \& Malina. Estos resultados pueden ayudar a los entrenadores a realizar la selección de las patinadoras en las disciplinas de parejas y danza.

Los valores del IMC presentados por las tres disciplinas, individual, parejas y danza (21,7, 20,8 y 21,4 respectivamente), son superiores a los presentados en el estudio de Monsma \& Malina (20,4, 19,6 y 19,3, para individual, pareja y danza respectivamente). Estos valores no coinciden con la literatura existente, pues enfatiza en la importancia que el aspecto estético y la linealidad tienen para el rendimiento en este deporte (Monsma \& Malina; Ross et al.; Vadocz et al.), por lo que cabría esperar valores inferiores de IMC. Pero es necesario remarcar que las edades de los estudios citados anteriormente son menores que los de la muestra de la presente investigación.

Las diferencias morfológicas presentadas por las tres disciplinas, confirman el diferente tipo de trabajo que realizan las patinadoras. Para las parejas existe mucho trabajo aéreo/acrobático que se enlaza a recepciones, por ello presenta valores inferiores en talla y envergadura, variables que benefician los momentos de inercia, y valores inferiores también en el perímetro del glúteo y medial del muslo, y superiores en el diámetro biestiloideo, estas diferencias estadísticas recalcan la importancia del agarre y el lanzamiento de su cuerpo por parte de la pareja. Para las patinadoras de danza, su trabajo se centra en desplazamientos y equilibrios coordinados con su pareja sin fase aérea, por ello, valores elevados de envergadura, de perímetros (brazo relajado, glúteo y medial de la pierna), del diámetro biestiloideo y valores bajos de endomorfia favorecen la realización de grandes desplazamientos agarradas a su pareja, reforzando las características de la linealidad.

El somatotipo de las patinadoras de parejas y danza (mesomórfico balanceado) es diferente de las patinadoras de libre (endo-mesomórfico). Estos datos, excepto para la disciplina de danza, no coinciden con los expresados por diversos autores (Monsma \& Malina; Ross et al.; Vadocz et $a l$.), puesto que indican que la ectomorfia se relaciona con el rendimiento y la endomorfia no. Como expuso Ross $e t$ al., el aumento de la endomorfia puede ser una desventaja para patinar con agilidad, pero se compensa con la experiencia. En la interpretación de estos resultados, hay que tener presente varios aspectos. En primer lugar la edad de la muestra, y valorar que con el final del desarrollo, en la mujer existe tendencia a la endomorfia (Malina \& Bouchard; Ross et al.). En segundo lugar, hay que tener presente el tamaño de la muestra, lo que va en detrimento de la homogeneidad de las patinadoras.

En conclusión, a pesar de las categorías, no existen diferencias entre la estructura corporal de las patinadoras. La mesomorfía es la característica predomínate en todos los somatotipos analizados. El aspecto morfológico es muy importante en la selección de las patinadoras, más concretamente para las disciplinas de pareja y danza.

\section{AGRADECIMIENTOS}

Los autores agradecen a todas las personas que han hecho posible la realización de este trabajo, especialmente a la Organización del 51 Campeonato del Mundo de Patinaje Artístico Sobre Ruedas, a la Federación Española de Patinaje, al cuerpo técnico de cada país participante y a todos los deportistas que participaron en el estudio.

VILA, M. H.; MANCHADO, C. \& FERRAGUT, C. Anthropometry, body composition and somatotype characteristics of world class female roller skaters by discipline. Int. J. Morphol., 33(3):1130-1135, 2015.

SUMMARY: The aim of the study was to describe and compare anthropometric characteristics, body composition and somatotype of female roller skaters by levels (Junior and Senior) and by disciplines (freestyle, pairs and dance). Sixty-two world class female roller skaters were assessed (36 freestyle roller skaters, 13 dance roller skaters and 13 pair roller skaters). A one-way analysis of variance (ANOVA) was used to determine if significant differences existed in the anthropometric profile among skating discipline and a student $t$ test in order to assess differences between junior and senior levels. There are no significant differences between junior and senior roller skaters. Junior female roller skaters show an endo-mesomorphic somatotype and senior female roller skaters show a balanced mesomorphic somatotype. Roller dance skaters and roller pair skaters show a balanced mesomorphic somatotype and roller freestyle skaters show an endo-mesomorphic somatotype. This paper, reports specific reference data for world class female roller skaters.

KEY WORDS: Women; Elite; Dance roller skaters; Freestyle roller skaters; Pair roller skaters; Fat percentage. 


\section{REFERENCIAS BIBLIOGRÁFICAS}

Brown, J. Sports Talent: How to identify and develop outstanding athletes. Champaign, Human Kinetics, 2001.

Carter, J. E. The somatotypes of athletes--a review. Hum. Biol., 42(4):535-69, 1970.

Carter, J. E. L. Physical Structure of Olympic Athletes. Part I: The Montreal Olympic Games Anthropological Project. 7: Body composition of Montreal Olympic Athletes. En: Jokl, E. \& Hebbelinck, M. Medicine and Sport. Vol. 16. New York, S. Karger, 1982. pp.107-16.

Carter, J. E. L. \& Heath, B. H. Somatotyping - development and applications. New York, Cambridge University Press, 1990.

Delistraty, D. A.; Reisman, E. J. \& Snipes, M. A physiological and nutritional profile of young female figure skaters. J. Sports Med. Phys. Fitness, 32(2):149-55, 1992.

Faulkner J. Physiology of swimming and diving. En: Falls, H. (Ed.). Exercise Physiology. Baltimore, Academic Press, 1968.

Kramer, C. Y. Extension of multiple range test to group means with unequal numbers of replications. Biom., 12(3):307-10, 1956.

Leone, M.; Lariviere, G. \& Comtois, A. S. Discriminant analysis of anthropometric and biomotor variables among elite adolescent female athletes in four sports. J. Sports Sci., 20(6):443-9, 2002.

Malina, R. M.; Bouchard, C. \& Bar-Or, O. Growth, maturation, and physical activity. 2nd ed. Champaign, Human Kinetics, 2004.

Monsma, E. V. Puberty and physical self-perceptions of competitive female figure skaters II: maturational timing, skating context, and ability status. Res. Q. Exerc. Sport, 79(3):411-6, 2008.

Monsma, D. V. \& Malina, R. M. Anthropometry and somatotype of competitive female figure skaters 11-22 years. Variation by competitive level and discipline. J. Sports Med. Phys. Fitness, 45(4):491-500, 2005.

Niinimaa, V. Figure skating: What do we know about it? Phys. Sports Med., 10(1):51-6, 1982.

Norton, K. \& Olds, T. Morphological evolution of athletes over the 20th century: causes and consequences. Sports Med., 31(11):763-83, 2001.

Podolsky, A.; Kaufman, K. R.; Cahalan, T. D.; Aleshinsky, S. Y. \& Chao, E. Y. The relationship of strength and jump height in figure skaters. Am. J. Sports Med., 18(4):400-5, 1990.
Ross, W. D.; Brown, S. R.; Yu, J. W. \& Faulkner, R. A. Somatotype of Canadian figure skaters. J. Sports Med. Phys. Fitness, 17(2):195-205, 1977.

Stewart, A.; Marfell-Jones, M.; Olds, T. \& Ridder, H. International Standards for anthropometric assessment. Lower Hutt, International Society for the Advancement of Kinanthropometry (ISAK), 2011.

Vadocz, E. A.; Siegel, S. R. \& Malina, R. M. Age at menarche in competitive figure skaters: variation by competency and discipline. J. Sports Sci., 20(2):93-100, 2002.

Whisenhunt, B. L.; Williamson, D. A.; Drab-Hudson, D. L. \& Walden, H. Intervening with coaches to promote awareness and prevention of weight pressures in cheerleaders. Eat Weight Disord., 13(2):102-10, 2008.

Ziegler, P. J.; Jonnalagadda, S. S. \& Lawrence, C. Dietary intake of elite figure skating dancers. Nutr. Res., 21(7):983-92, 2001.

Dirección para Correspondencia:

María Helena Vila Suárez

Faculty of Education and Sport Science

University of Vigo

Pontevedra

SPAIN

Email: evila@uvigo.es

Recibido : 05-12-2014

Aceptado: 17-07-2015 